evaluation as potential anti-gametocyte vaccine candidates and/ or biomarkers for gametocyte carriage.

\title{
PA-050 ANTIBODY RESPONSES TO SURFACE ANTIGENS OF PLASMODIUM FALCIPARUM GAMETOCYTE-INFECTED ERYTHROCYTES AND THEIR RELATION TO GAMETOCYTAEMIA
}

Bismarck Dinko, ${ }^{1}$ Elizabeth King, ${ }^{2}$ Geoffrey Targett, ${ }^{2}$ Colin Sutherland ${ }^{2} .{ }^{1}$ UHAS, Ghana; ${ }^{2}$ LSHTM, United Kingdom

\subsection{6/bmjgh-2016-000260.83}

Background An essential element for continuing transmission of Plasmodium falciparum is the availability of mature gametocytes in human peripheral circulation for uptake by mosquitoes. Natural immune responses to circulating gametocytes may play a role in reducing transmission from humans to mosquitoes.

Methods Here, antibody recognition of the surface of mature intra-erythrocytic gametocytes produced either by a laboratory-adapted parasite, $3 \mathrm{D} 7$, or by a recent clinical isolate of Kenyan origin (HL1204), was evaluated longitudinally in a cohort of Ghanaian school children by flow cytometry.

Results This showed that a proportion of children exhibited antibody responses that recognised gametocyte surface antigens on one or both parasite lines. A subset of the children maintained detectable anti-gametocyte surface antigen (GSA) antibody levels during the five week study period. There was indicative evidence that children with anti-GSA antibodies present at enrolment were less likely to have patent gametocytaemia at subsequent visits $(\mathrm{OR}=0.29,95 \% \mathrm{CI}: 0.06-1.05 ; \mathrm{p}=0.034)$.

Conclusions Our data support the existence of antigens on the surface of gametocyte - infected erythrocytes, but further studies are needed to confirm whether antibodies against them reduce gametocyte carriage. The identification of GSA would allow their 